\title{
QUALIDADE DO AR INTERNO EM AMBIENTES CLIMATIZADOS - VERIFICAÇÃO DOS PARAMETROS FÍSICOS E CONCENTRAÇÃO DE DIÓXIDO DE CARBONO EM ẢGÊNCIA BANCÁRIA
}

\author{
Autores: Waldir Nagel Schirmer*, Mariani Silvia Ester Szymanski, Mayara Ananda Gauer
}

Curso de Engenharia Ambiental da Universidade Estadual do Centro-oeste, Irati-PR, Brazil.

*E-mail: wanasch@yahoo.com.br

Recebido em 28 de janeiro de 2009 Aceito em 20 de junho de 2009

\section{RESUMO}

A presença de poluentes químicos e biológicos no ar interno de estabelecimentos públicos cria condições que podem comprometer a saúde e produtividade dos funcionários. Assim, estes estabelecimentos necessitam de sistemas de climatização bem projetados, que forneçam taxas de ventilação adequadas para garantir o conforto e bem-estar de seus ocupantes, bem como a assepsia dos ambientes. O objetivo deste trabalho foi avaliar a qualidade do ar interno (QAI) de um banco da região centro-sul do Paraná, através da verificação dos parâmetros físicos de conforto (temperatura, umidade relativa e velocidade do ar) e das concentrações de dióxido de carbono, seguindo-se a metodologia recomendada pela Resolução $\mathrm{n}^{\circ} 09$ da Agência Nacional de Vigilância Sanitária (ANVISA). Os resultados obtidos permitiram afirmar que a QAI dos ambientes estudados está comprometida, já que alguns dos parâmetros avaliados apresentaram valores superiores aos recomendados pela referida norma. As altas concentrações de $\mathrm{CO}_{2}$ obtidas nestes ambientes podem ser justificadas pela falta de renovação de ar, uma vez que, os aparelhos de climatização destes ambientes são desprovidos desta função. Sugere-se que, para que se verifiquem melhorias na QAI, efetuem-se mudanças nos sistemas de climatização, fazendo-se uso daqueles projetados especialmente para tais tipos de ambientes e que contemplem a renovação do ar a taxas aceitáveis pela legislação vigente.

Palavras-chave: Agência bancária; Dióxido de carbono; Qualidade do ar interno.

\section{INTRODUÇÃO}

Entende-se por ar interno aquele de áreas não industriais, como habitações, escritórios, escolas e hospitais [1]. O estudo de sua qualidade é importante para garantir a saúde dos ocupantes, bem como o ótimo desempenho de suas atividades [2].

De acordo com padrões da Organização Mundial de Saúde (OMS), mais da metade dos locais fechados como empresas, escolas, cinemas, residências e até hospitais têm ar de má qualidade. Essa baixa qualidade é causada, principalmente, pela má higienização dos aparelhos de ar condicionado e pela falta de controle periódico sobre as possíveis fontes de contaminação [3].

Sabendo-se que o ar condicionado, quando não devidamente operado, pode ser causador de inúmeros problemas à saúde e ao conforto, considera-se que este seja de elevada importância na manutenção de condições favoráveis ou desfavoráveis em ambientes climatizados. Sendo assim, o objetivo da climatização é o tratamento do ar para controle da temperatura, umidade e pureza, visando atender às necessidades do ambiente [4].

Segundo Jones [4], admite-se que a ventilação seja um dos principais fatores que interferem na qualidade do ar interno. A ventilação é definida como a combinação de processos que resultam no fornecimento de ar externo e retirada do ar viciado (carregado de poluentes) de dentro do edifício. Basicamente os processos que envolvem a ventilação são a entrada do ar externo, o condicionamento e mistura do ar por todo o edifício seguido de sua exaustão. Assim, para se ter um edifício saudável, deve-se ter uma boa qualidade do ar interior, através do uso de adequadas taxas de ventilação, de sistemas de automação predial e principalmente de um monitoramento contínuo dessas instalações [5].

Graudenz et al [6], com o intuito de descrever e comparar indicadores de renovação de ar realizaram medições de dióxido de carbono $\left(\mathrm{CO}_{2}\right)$ em bancos, unidades de saúde, escritórios, hotéis, indústrias e outros locais, em todas as regiões do Brasil, num período de seis anos, totalizando 17.885 medições em ambientes internos. Os resultados obtidos indicaram os maiores níveis médios de $\mathrm{CO}_{2}$ em bancos (848,48 ppm), seguidos pelas unidades de saúde - hospitais e laboratórios (775,13 ppm). Outros trabalhos, todos realizados em locais de grande circulação pública (como escolas, hospitais, shoppings, bancos, etc.), foram realizados por [7-11]. Em todos eles, os níveis dos principais parâmetros de controle de ambientes internos (como $\mathrm{CO}_{2}$ ou microbiológicos) foram seguidamente ultrapassados, apontando uma fragilidade do sistema de ventilação desses ambientes em manter condições razoáveis de salubridade a seus ocupantes. Gupta et al. [11] observaram claramente que as concentrações de $\mathrm{CO}_{2}$ podem 
ser utilizadas como parâmetro-indicador da qualidade do ar interno (QAI), uma vez que vários sintomas relacionados à síndrome de edifícios doentes (SED) como irritação dos olhos, garganta, cansaço, letargia, etc. estavam linearmente relacionados à concentração desse gás.

Tais resultados evidenciam a importância do monitoramento constante do ar desses ambientes, como forma de prevenir e evitar prejuízos à saúde humana bem como a redução na produtividade dos funcionários aí presentes.

Considerando-se a importância da QAI, este trabalho teve por objetivo avaliar as condições de conforto a partir da análise dos parâmetros físicos (temperatura, umidade relativa, velocidade e taxas de renovação do ar) e das concentrações de $\mathrm{CO}_{2}$ em uma agência bancária localizada na região centro-sul do Paraná.

\section{MATERIAL E MÉTODOS}

\subsection{Caracterização do local de estudo}

A agência bancária onde se realizou o presente estudo é construída em dois pavimentos de áreas equivalentes (de $468,15 \mathrm{~m}^{2}$ cada uma, conforme Figura 1). Como a ocupação dos pavimentos são variáveis, considerouse a taxa média de ocupação fornecida pela gerência, sendo 65 e 35 pessoas no primeiro e segundo pavimentos, respectivamente, incluindo funcionários e clientes.

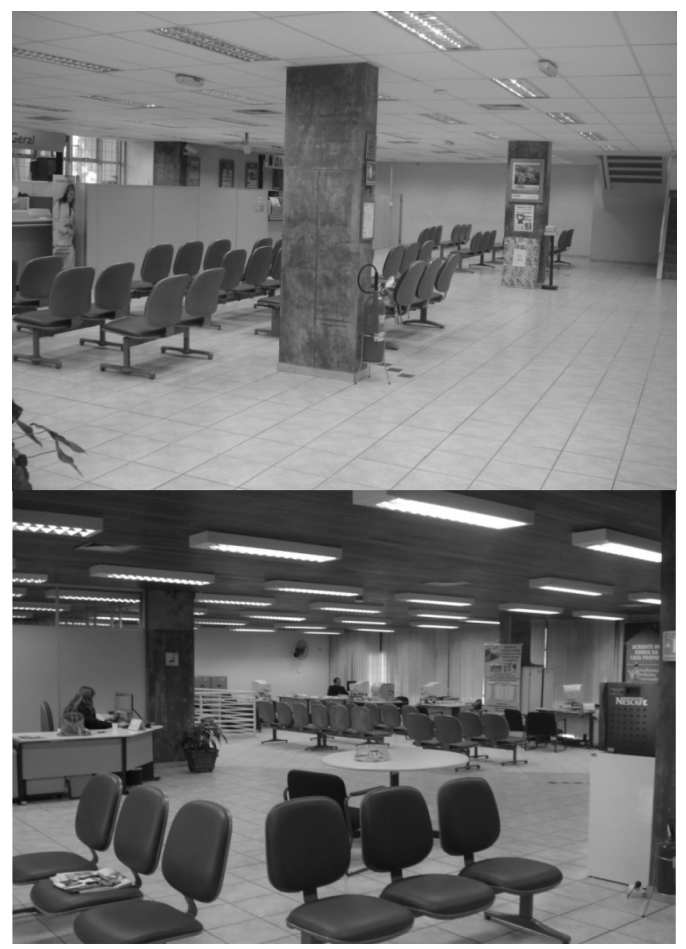

Figura 01 - Agência bancária onde se realizou o estudo: pavimentos 1 (acima) e 2 (abaixo)
A climatização da agência é feita utilizando-se aparelho de ar condicionado do tipo "self contained". Estes aparelhos são condicionadores de ar compactos onde todos os componentes necessários ao tratamento do ar, como filtragem, umidificação, refrigeração e movimentação do ar estão contidos no gabinete (Figura 2). O processo de condensação em aparelhos "self contained" pode ser realizado utilizando-se ar ou água.

No caso do aparelho utilizado na agência, a condensação é feita por ar e a distribuição do ar é realizada por redes de dutos. Projetado em peça única, esse sistema permite o escoamento do líquido condensado, evitando os desconfortos causados pela estagnação da água e formação de mofos. Segundo o fabricante, sua utilização é indicada em ambientes onde há elevada ocupação ou necessidade de alta qualidade de ar insuflado, apresentando como vantagens o reduzido custo de operação, eficiência no controle das condições ambientais e pouca área requerida para sua instalação [12-13]. Cabe destacar que no caso da agência avaliada não ocorrem ciclos de renovação do ar, apenas recirculação.

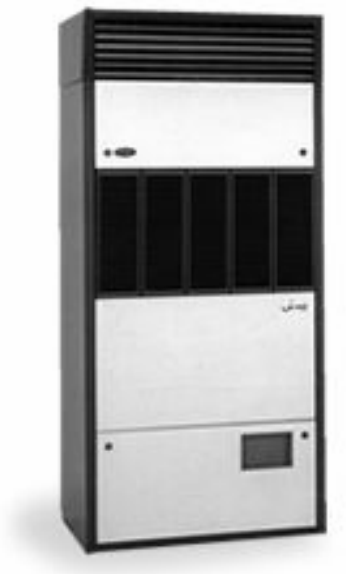

FIGURA 02 - Modelo do ar condicionado utilizado na agência - "self contained" [12].

\subsection{Determinação das concentrações de $\mathrm{CO}_{2}$ e parâmetros físicos}

Para determinação da concentração de dióxido de carbono, a metodologia selecionada foi a sugerida pela Resolução ANVISA nº 09, de 16 de janeiro de 2003 (Norma Técnica 002) [14]. Para tanto, utilizou-se o analisador automático de $\mathrm{CO}_{2}$ modelo $\mathrm{C}-02$, da marca Instrutherm, devidamente calibrado, o qual possibilita a leitura direta mediante a utilização de sensor infravermelho nãodispersivo. O referido sensor apresenta faixa de detecção de 0 a 6.000 ppm e resolução de 1 ppm.

O trabalho compreendeu três campanhas (uma por semana), realizadas nos dias 15 e 16 de abril ( $1^{\text {a }}$ campanha), 22 e 23 de abril ( $2^{\text {a }}$ campanha), e 29 e 30 de abril ( $3^{\text {a }}$ campanha) de 2008, sempre nos horários de pico da agência (por volta do meio dia). O número de amostragens em cada recinto foi definido segundo a mesma resolução (em função das áreas construídas), sendo realizadas três amostragens em cada pavimento da agência (em três pontos distintos por pavimento). $\mathrm{O}$ valor final considerado de cada amostragem 
constou de uma aritmética de dez medições (em cada ponto).

Para a medição da temperatura, umidade relativa e velocidade do ar, utilizou-se termoanemômetro de fio quente, da marca Lutron, modelo AM-420HHA. A amostragem foi realizada a $1,5 \mathrm{~m}$ de altura (em frente à saída de ar do aparelho), de acordo como o recomendado pela própria Resolução ANVISA.

\section{RESULTADOS E DISCUSSÃO}

3.1 Verificação dos parâmetros físicos - temperatura, umidade relativa, velocidade e taxas de renovação do ar

Segundo Basto [13], a qualidade do ar de um ambiente está diretamente relacionada ao conforto térmico dos ocupantes que, por sua vez, é determinado pelos sistemas de climatização. Sendo assim, para se estimar as condições de conforto a que os ocupantes estão expostos, costuma-se monitorar a temperatura, umidade relativa e velocidade do ar.

De acordo com a Resolução ANVISA no 09/03, recomenda-se que a temperatura de bulbo seco deve permanecer na faixa entre 23,0 e $26,0{ }^{\circ} \mathrm{C}$ no verão e entre 20,0 e $22,0^{\circ} \mathrm{C}$ durante o inverno. Essa faixa irá depender do local e da finalidade da instalação. Da mesma forma, admite-se que a umidade relativa do ar (UR) varie de 40 a $65 \%$ no verão e de 35 a $65 \%$ no inverno. Ainda segundo recomendações da referida resolução, a velocidade do ar a $1,5 \mathrm{~m}$ do piso deve ser de no máximo $0,25 \mathrm{~m} \mathrm{~s}^{-1}$.

Na Tabela 1 são apresentados os valores de temperatura e umidade relativa do ar obtidos durante a avaliação, destacando-se que os dados apresentados são referentes às médias aritméticas dos valores obtidos nas seis medições (dias) procedidas.

Durante as medições verificou-se a velocidade do ar, a 1,5 m de altura, de 0,07 e $0,15 \mathrm{~m} \mathrm{~s}^{-1}$ nos pavimentos $1 \mathrm{e}$ 2 , respectivamente. A partir da comparação destes dados com o estabelecido na Resolução, verifica-se que ambos os pavimentos atendem à legislação.

TABELA 1 - Médias dos valores de temperatura e umidade relativa do ar.

\begin{tabular}{clll}
\hline Data & Pavimento & $\begin{array}{l}\text { Temperatura } \\
\left({ }^{\circ} \mathrm{C}\right)\end{array}$ & $\begin{array}{l}\text { Umidade Relativa } \\
(\%)\end{array}$ \\
\hline \multirow{2}{*}{15 abr. } & 1 & 24,8 & 48 \\
& 2 & 25,3 & 50,8 \\
16 abr. & 1 & 23,7 & 65,5 \\
& 2 & 23,9 & 59,2 \\
22 abr. & 1 & 23,5 & 63,8 \\
& 2 & 24,5 & 58,2 \\
23 abr. & 1 & 23,4 & 57,6 \\
& 2 & 24,5 & 55,4 \\
29 abr. & 1 & 24,1 & 70,4 \\
& 2 & 25,9 & 62,3 \\
30 abr. & 1 & 23,6 & 42,1 \\
& 2 & 23,9 & 45,1 \\
\hline
\end{tabular}

Os valores das velocidades são equivalentes em todas as medições realizadas em cada pavimento. São resultado de média aritmética de 10 valores amostrados.
Nesse caso, pela tabela 1, vê-se que a recomendação para a temperatura ambiente não é atendida, haja vista que em todas as medições a temperatura verificada foi acima de $23,5{ }^{\circ} \mathrm{C}$ quando o recomendado é de, no máximo, 22,0 ${ }^{\circ} \mathrm{C}$. Quanto à UR, com exceção à verificada no dia 29 de abril no pavimento 1 , durante todo o período de amostragem verificou-se o atendimento ao prescrito em Resolução (35 a 65\%).

A Resolução ANVISA nº 09 estabelece taxas de renovação do ar em ambientes climatizados na ordem de 27 $\mathrm{m}^{3} \mathrm{~h}^{-1}$ pessoa ${ }^{-1}$ e $17 \mathrm{~m}^{3} \mathrm{~h}^{-1}$ pessoa ${ }^{-1}$ para locais com elevada rotação de pessoas. Nesse caso, com base nos valores apresentados na Tabela 2, pelo menos em termos quantitativos, verifica-se o atendimento à legislação no primeiro pavimento. No entanto, considerando-se que não há captação de ar externo, pode-se afirmar que essa taxa diz respeito apenas à recirculação do ar ambiente, não havendo, portanto, uma "renovação de ar" propriamente dita no ambiente.

TABELA 2 - Taxas de Renovação do ar verificadas.

\begin{tabular}{lll}
\hline & $\begin{array}{l}\text { TABELA 2 - Taxas de Renovação do ar verificadas. } \\
1\end{array}$ & $\begin{array}{l}\text { Pavimento } \\
2\end{array}$ \\
\hline Número de difusores & 12 & 16 \\
Taxa de entrada de $\operatorname{ar}\left(\mathrm{m}^{3} \mathrm{~h}^{-1}\right)$ & 1130,48 & 557,81 \\
$\begin{array}{l}\text { Número de Pessoas } \\
\text { Taxa de Ar/pessoa }\left(\mathrm{m}^{3} \text { pessoa }\right.\end{array}$ & 65 & 35 \\
$\left.1^{1}\right)$ & 17,39 & 15,94 \\
\hline
\end{tabular}

Assim, embora muitas vezes os valores de trocas de ar estejam dentro do recomendado, apenas a análise da concentração do $\mathrm{CO}_{2}$ vai apontar se essa troca é suficiente para a renovação do mesmo.

\subsection{Verificação das concentrações de $\mathrm{CO}_{2}$}

De acordo com a ACGIH [15], apesar de exposições aos níveis comumente encontrados em ambientes interiores (350 a 2500 ppm) não serem considerados causa direta de efeitos à saúde, evidenciam-se muitos impactos diretos e mensuráveis na saúde humana. Sabe-se, por exemplo, que a baixa renovação de ar pode resultar em sonolência e perda de produtividade até mesmo em indivíduos saudáveis.

Segundo recomendações da Resolução ANVISA $n^{\circ}$ 09, a concentração de $\mathrm{CO}_{2}$ em ambientes interiores não deve ultrapassar $1000 \mathrm{ppm}$. Ao se proceder à comparação dos níveis medidos (Tabela 3) com a legislação vigente (concentração limite de $\mathrm{CO}_{2}$ ) nota-se que, no pavimento 1 , apenas a primeira medição excedeu o limite permitido, enquanto que no segundo pavimento, das seis medições feitas, quatro excederam os limites permitidos pela legislação. As concentrações elevadas de $\mathrm{CO}_{2}$ podem ser explicadas pelo fato de que o sistema de ar condicionado utilizado promove apenas uma recirculação do ar ambiente, sem realizar captação do ar externo.

A partir da análise da Tabela 3 verifica-se que as concentrações de $\mathrm{CO}_{2}$ no pavimento 2 durante o período de análise apresentam níveis mais elevados em relação ao pavimento 1 em todas as medições realizadas. 
TABELA 3 - Médias dos parâmetros avaliados no estudo

\begin{tabular}{|c|c|c|c|c|c|c|c|}
\hline \multirow{2}{*}{ Pav. } & \multirow{2}{*}{ Concentração de $\mathrm{CO}_{2}(\mathrm{ppm})$} & \multicolumn{6}{|c|}{ Período de Avaliação } \\
\hline & & $15 / 04 / 08$ & $16 / 04 / 08$ & $22 / 04 / 08$ & $23 / 04 / 08$ & $29 / 04 / 08$ & $30 / 04 / 08$ \\
\hline \multirow{2}{*}{1} & Média & 1015,17 & 950,90 & 956,75 & 944,18 & 799,22 & 559,80 \\
\hline & Desvio padrão & 19,31 & 31,66 & 45,86 & 100,00 & 10,35 & 55,68 \\
\hline \multirow{2}{*}{2} & Média & 1537,43 & 1076,05 & 1240,29 & 1318,96 & 989,93 & 727,80 \\
\hline & Desvio padrão & 50,51 & 21,34 & 49,15 & 78,43 & 22,86 & 50,86 \\
\hline
\end{tabular}

As concentrações de $\mathrm{CO}_{2}$ apresentadas equivalem à média aritmética (geral) dos três pontos (locais) amostrados (total de 30 medições por pavimento).

Considerando-se que no segundo pavimento a ocupação é menor em relação ao primeiro, as concentrações elevadas podem ser justificadas devido ao fato de que, por se localizar no segundo piso, a única entrada de ar, além daquele insuflado pelo ar condicionado, é proveniente do acesso do primeiro piso.

De acordo com a NBR 6401 - NB 10 [16], portas giratórias utilizadas em agências bancárias permitem uma infiltração média de ar de $11 \mathrm{~m}^{3} \mathrm{~h}^{-1}$ pessoa $^{-1}$. Sendo assim, uma menor concentração de $\mathrm{CO}_{2}$ no pavimento 1 também pode ser explicada por esta entrada de ar, a qual é responsável por promover uma diluição considerável do ar ambiente. Outra explicação pode ser uma melhor performance de renovação de ar proporcionada pelos aparelhos desse andar em relação aos do segundo.

\section{CONCLUSÕES}

Do ponto de vista dos parâmetros físicos, verificase uma conformidade destes com os valores especificados pela normalização específica, com exceção das temperaturas. Estes dados podem ser utilizados para análise da qualidade do ar do aspecto de temperatura, diluição e conforto térmico, uma vez que são influenciados apenas pela eficiência dos aparelhos de climatização.

Verificou-se que em $67 \%$ das amostragens realizadas no segundo pavimento as concentrações de $\mathrm{CO}_{2}$ estavam acima do permitido na legislação, o que evidencia a necessidade da captação de ar externo de boa qualidade. Além disso, nota-se que não houve correlação entre a ocupação e os níveis verificados de $\mathrm{CO}_{2}$, o que indica que o próprio sistema atual de climatização não está sendo suficiente para garantir uma renovação adequada do ar ambiente.

\section{INDOOR AIR QUALITY IN CLIMATIZED} ENVIRONMENTS - VERIFICATION OF PHYSICAL PARAMETERS AND CARBON DIOXIDE IN BANK AGENCIES.

ABSTRACT: The presence of chemical and biological pollutants in the indoor air of public buildings creates conditions that may prejudice the health and productivity of employees. Thus, these places need air conditioning well designed, to provide adequate ventilation rates to ensure the comfort of its occupants and the aseptic of environments. The objective of this study was to evaluate the indoor air quality (IAQ) in a bank located in south-central region of Parana state, Brazil, by checking the physical parameters of comfort (temperature, relative humidity and average air speed) and the concentrations of carbon dioxide, following the procedure recommended by Resolution No. 09 of the National Sanitary Surveillance Agency (ANVISA). The results showed that the IAQ in those environments was compromised, once some of the parameters showed values higher than those recommended by that resolution. High concentrations of $\mathrm{CO}_{2}$ obtained can be justified by the lack of renewal of air. It is suggested that, for improvements occurring in the IAQ, the air conditioning systems must to be substituted for those especially designed for that environment and that allowed the renewal of the air at rates acceptable to the existing legislation.

Keywords: Bank agency; Carbon dioxide; Indoor air quality.

\section{REFERÊNCIAS}

[1] WANG, S.; ANG, H. M.; TADE, M. O.; Environ. Intern., Vol. 33, p. [1] WANG, S.; ANG, H. M.; TADE, M. O.; Environ. Intern., Vol. 33, p. 694 705, 2007.

[2] GIODA, A.; AQUINO NETO, F. R.; Caderno da Saúde Pública, Vol. 19, p. 1389-1387, 2003.

[3] LIMA DE PAULA, J. F. Aeromicrobiota do ambiente cirúrgico: princípios e peculiaridades da climatização artificial. 2003. $111 \mathrm{f}$. Dissertação (Mestrado em Enfermagem Fundamental) - Universidade de São Paulo, São Paulo. 2003.

[4] JONES, A.P.; Atmos. Environ., Vol. 33, p. 4535-4564, 1999.

[5] CARMO, A. T.; PRADO, R. T. A.; Qualidade do Ar Interno; EPUSP; São Paulo, 1999. 35 p.

[6] GRAUDENZ, G.S.; DANTAS, E.; PEREIRA, A.C.; SENSOUSSAN, M.D.; RICARD, I.; GURGEL, M.; TRIBESS, A. Indicadores de renovação do ar em ambientes climatizados - a experiência brasileira. Disponível em: http://www.nalco.com/PDF/Brazil/indicadores-renovacao.pdf. Acesso em 13 abr. 2009.

[7] NORBÄCK, D.; SMEDJE, G.; Ann. Occup. Hygien., Vol. 41, Supplement 01, p. 689-694, 1997. 
[8] LI, W-M.; LEE, S. C.; CHAN, L. Y.; Science of Total Environment. Vol. 273, p. 27-40, 2001.

[9] WONG, N. H.; HUANG, B.; Building and Environment, Vol. 39, p. 1115-1123, 2004

[10] LEE, S. C.; CHAN, L. Y.; CHIU, M. Y.; Envir. Intern., Vol. 14 n. 04, p. 443-450, 1999.

[11] GUPTA, S.; KHARE, M.; GOYAL, R.; Build. and Envir., Vol. 42, p. 2797-2809, 2007.

[12] SPRINGER - CARRIER. Catálogo Técnico Self New Generation. 2004. $36 \mathrm{p}$.

[13] BASTO, J. E. Requisitos para Garantia da Qualidade do Ar em Ambientes Climatizados Enfoque em Ambientes Hospitalares. 2005. 110f. Monografia (Pós Graduação em Engenharia de Segurança do Trabalho) Universidade Federal de Santa Catarina, Florianópolis. 2005.

[14] BRASIL. Ministério da Saúde. 2003. Agência Nacional de Vigilância Sanitária (ANVISA). Resolução nº 9, de 16 de janeiro de 2003.

[15] ACGIH - American Conference of Governmental Industrial Hygienists. 1991. Documentation of the threshold limit values and biological exposure indices. 6th edition, Cincinnati-Ohio.

[16] ABNT - Associação Brasileira de Normas Técnicas. 1980. NBR 6401 NB 10: Instalações centrais de ar-condicionado para conforto - Parâmetros básicos de projeto. ABNT: Rio de Janeiro, 17p. 